\title{
Lower Bounds for Generalized Quantum Finite Automata
}

\author{
Mark Mercer \\ Département d'Informatique, \\ Université de Sherbrooke, QC, Canada
}

\begin{abstract}
We obtain several lower bounds on the language recognition power of Nayak's [12] generalized quantum finite automata (GQFA). Techniques for proving lower bounds on Kondacs and Watrous' one-way quantum finite automata (KWQFA) were introduced by Ambainis and Freivalds [2], and were expanded in a series of papers. We show that many of these techniques can be adapted to prove lower bounds for GQFAs. Our results imply that the class of languages recognized by GQFAs is not closed under union. Furthermore, we show that there are languages which can be recognized by GQFAs with probability $p>1 / 2$, but not with $p>2 / 3$.
\end{abstract}

Quantum finite automata (QFA) are online, space-bounded models of quantum computation. Similar to randomized finite automata [16] where the state is a random variable over a finite set, the state of a QFA is a quantum superposition of finite dimension. The machine processes strings $w \in \Sigma^{*}$ by applying a sequence of state transformations specified by the sequence of letters in $w$, and the output of the machine is determined by a measurement of the machine state. A central problem is to characterize the language recognition power of QFAs.

Most QFA research has been focused on the case where the transformations are limited to various combinations of unitary transformations and projective measurements. The class of languages recognized by these QFAs is a strict subset of the regular languages, so QFAs are less powerful than their classical counterparts. This is due to the fact that, unlike many other models of quantum computation such as quantum Turing machines [6] or quantum circuits [17], QFAs lack the linear space overhead which is required to convert classical computation into reversible computation [5]. However, there are languages which can be recognized by QFAs using exponentially fewer states than the smallest deterministic or randomized finite automaton $[2,7]$.

The simplest type of QFA is the measure-once QFA (MOQFA) model of Moore and Crutchfield [11]. These QFAs are limited to recognizing those languages whose minimal automaton is such that each letter induces a permutation on the states. Two types of generalizations of the MOQFA model have been considered. In the first type, the machine is allowed to halt before reading the entire input word. This corresponds to Kondacs and Watrous' one-way QFAs (KWQFAs) [10]. The second type is to allow state transformations to include 
the application of quantum measurements, which generates some classical randomness in the system. This corresponds to Ambainis et. al's Latvian QFAs (LQFAs) [1].

Nayak [12] investigated a model called generalized QFAs (GQFAs), which generalize both KWQFAs and LQFAs. This paper introduced new entropy-based techniques which were used to show that GQFAs cannot recognize the language $\Sigma^{*} a$. These techniques have since been used to obtain lower bounds on quantum random access codes [12] and quantum communication complexity [13]. However, no further lower bounds have been shown for GQFAs.

In a series of papers $[2,8,4,3]$, a number of important lower bounds on the power of KWQFA were identified. The main tool used in these results was a technical lemma which is used to decompose the state space of a KWQFA into two subspaces (called the ergodic and transient subspaces) in which the state transitions have specific behaviors. In this paper, we show that this lemma can be adapted to the case of GQFA. The framework of our proof follows the basic outline of [2], however we must overcome a number of technical hurdles which arise from allowing classical randomness in the state.

We use this lemma to highlight a number of relevant properties of the class of languages recognized by GQFA. Following [4], we can use the lemma to show that a certain property of the minimal automaton for $L$ implies that $L$ is not recognizable by a GQFA. We use this result to show that the class of languages recognized by this model is not closed under union. Furthermore, we show the existence of languages which can be recognized by GQFA with probability $p=$ $2 / 3$ but not $p>2 / 3$. These results highlight the key similarities and differences between KWQFA and GQFA.

The paper is organized as follows. In Section 2 we give definitions and basic properties of GQFA and we review the necessary background. In Section 3 we will state our main results. In Section 4 we prove the main technical lemma and in Section 5 we apply this lemma to prove the remaining results. In the last section we discuss open problems and future work.

\section{Introduction}

Let us review some concepts from quantum mechanics. See e.g. [14] for more details on the mathematics of quantum computation. We use the notation $|\psi\rangle$ to denote vectors in $\mathbb{C}^{n}$, and we denote by $\langle\psi|$ the dual of $|\psi\rangle$.

Let $Q$ be a finite set with $|Q|=n$, and let $\{|q\rangle\}_{q \in Q}$ be an orthonormal basis for $\mathbb{C}^{n}$. Then a superposition over $Q$ is a vector $|\psi\rangle=\sum_{q} \alpha_{q}|q\rangle$ which satisfies

$\langle\psi \mid \psi\rangle=\sum_{q}\left|\alpha_{q}\right|^{2}=1$. We say $\alpha_{q}$ is the amplitude with which $|\psi\rangle$ is in state $q$. The state space of a QFA will be a superposition over a finite set $Q$.

We consider two types of operations on superpositions. First, a unitary transformation $U$ is a linear operator on $\mathbb{C}^{n}$ such that the conjugate transpose $U^{\dagger}$ of $U$ satisfies $U^{\dagger} U=U U^{\dagger}=I$. Unitary operators are exactly those which preserve the inner product, thus unitary matrices map superpositions to superpositions. The second type of operation is projective measurements. Such measurements 
are specified by a set $\mathcal{M}=\left\{P_{i}\right\}$ of orthonormal projectors on $\mathbb{C}^{n}$ satisfying $\sum_{i} P_{i}=I$. The outcome of is the measurement $\mathcal{M}$ on state $|\psi\rangle$ is the random variable which takes the value $i$ with probability $\| P_{i}|\psi\rangle \|^{2}$. If the outcome of the measurement is $i$, the state is transformed to $\left|\psi^{\prime}\right\rangle=P_{i}|\psi\rangle / \| P_{i}|\psi\rangle \|$. Note that measurement induces a probabilistic transformation the state. Measurements describe the interface by which we obtain observations from a quantum system, but they also model decoherence, the process by which a quantum system becomes a probabilistic system through interaction with the environment (c.f. Chapter 8 of [14]).

A generalized QFA (GQFA) [12] is given by a tuple of the form:

$$
M=\left(\Sigma, Q, q_{0},\left\{U_{a}\right\}_{a \in \Gamma},\left\{\mathcal{M}_{a}\right\}_{a \in \Gamma}, Q_{a c c}, Q_{r e j}\right) .
$$

The set $\Sigma$ is the input alphabet. The working alphabet will be $\Gamma=\Sigma \cup\{\phi, \$\}$. The set $Q$ is finite set of state indices with $q_{0} \in Q, Q_{a c c}, Q_{r e j} \subseteq Q$. On input $w \in \Sigma^{*}, M$ will process the letters of the string $₫ w \$$ from left to right. The $\mathbb{\phi}$ and $\$$ characters are present to allow for pre- and post- processing of the state. The sets $\left\{U_{a}\right\}_{a \in \Gamma}$ and $\left\{\mathcal{M}_{a}\right\}_{a \in \Gamma}$ are collections of unitary transformations and projective measurements.

The state of the machine is expressed as a superposition over $Q$, and the initial state is $\left|q_{0}\right\rangle$. when a letter $a \in \Gamma$ is read, a state transformation is made in the manner we describe below. After each letter is read, the machine may decide to halt and accept the input, to halt and reject the input, or to continue processing the string. The set $Q$ is partitioned into three parts: an accepting set $\left(Q_{a c c}\right)$, a rejecting set $\left(Q_{r e j}\right)$ and a nonhalting set $\left(Q_{n o n}=Q-Q_{a c c} \cup Q_{r e j}\right)$. We define $P_{a c c}=\sum_{q \in Q_{a c c}}|q\rangle\langle q|$ and we likewise define $P_{r e j}$ and $P_{n o n}$. Finally, we define $\mathcal{M}_{\mathcal{H}}=\left\{P_{a c c}, P_{\text {rej }}, P_{\text {non }}\right\}$.

Suppose that after reading some input prefix the machine is in state $|\psi\rangle$. To process $a \in \Gamma$, we first apply the unitary $U_{a}$, then the measurement $\mathcal{M}_{a}$ (recall that this is a probabilistic transformation), then the measurement $\mathcal{M}_{H}$. If the outcome of the measurement $\mathcal{M}_{H}$ is acc or rej, then the machine halts and accepts or rejects accordingly. Otherwise, the outcome of the $\mathcal{M}_{H}$ was non and the machine reads the next symbol in the string ${ }^{1}$.

The GQFA defined above will behave stochastically. We will be interested in what languages can be recognized by this machine with bounded error. For $p>\frac{1}{2}$ we say that language $L \subseteq \Sigma^{*}$ is recognized by $M$ with probability $p$ if all words are correctly distinguished with probability at least $p$. We say that $L$ is recognized with bounded error if there is a $p>\frac{1}{2}$ such that $L$ is recognized with probability $p$.

Here are some basic facts about GQFAs. For all $p$, the class of languages recognized by GQFA with probability $p$ is closed under complement, inverse

\footnotetext{
1 The original definition allowed $\ell$ alternations of unitary operators and measurements per letter. However, such alternations can be simulated by a single transformation and measurement (Claim 1 of [1]) and so this change does not limit the class of transformations allowed by GQFAs.
} 
morphisms, and word quotient. We also make note of the relationship between GQFAs and other QFA definitions. Firstly, in the case that each $\mathcal{M}_{a}$ is equal to the trivial measurement $\{I\}$, then we obtain KWQFAs as a special case. Second, in the case that we are promised that the machine does not halt until the entire input is read, then we have the special case of Ambainis et al's LQFAs. If both of these conditions hold, we obtain MOQFAs.

In this paper we will see that many of the lower bounds for KWQFAs apply also to GQFAs. It should be noted, however, that GQFA are strictly more powerful than KWQFA. In [1] it was shown that any language $L$ whose transition monoid is a block group [15] can be recognized by an LQFA with probability $1-\varepsilon$ for any $\varepsilon>0$. This language class corresponds exactly to the boolean closure of languages of the form $L_{0} a_{1} L_{1} \ldots a_{k} L_{k}$, where the $a_{i}$ 's are letters and the $L_{i}$ 's are recognized by permutation automata. On the other hand, KWQFA cannot recognize $\Sigma^{*} a \Sigma^{*} b \Sigma^{*}$ with probability more than $7 / 9$ [2]. It was furthermore shown in [1] that LQFA cannot recognize the languages $a \Sigma^{*}$ or $\Sigma^{*} a$. We will need these properties in order to prove our results.

Furthermore it is known that KWQFA, and hence GQFA, can recognize languages which cannot be recognized by LQFA. For example KWQFA can simulate a certain type of reversible automaton where $\delta\left(q_{1}, x\right)=\delta\left(q_{2}, x\right)=q_{2}$ is permitted only when $q_{2}$ is a sink. These machines, and class of languages which they recognize, were considered in [9]. They include the language $a \Sigma^{*}$, which is not recognized by LQFA.

Finally, a few notes about density matrices. Recall the state of a GQFA after reading some input prefix is a random variable. In other words, the state is taken from a probability distribution $\mathcal{E}=\left\{\left(p_{j},\left|\psi_{j}\right\rangle\right)\right\}$ of superpositions, where $\left|\psi_{j}\right\rangle$ occurs with probability $p_{j}$. Such systems are called mixed states. The measurement statistics which can be obtained from measuring a mixed state can be described succinctly in terms of density matrices. The density matrix corresponding to $\mathcal{E}$ is $\rho=\sum_{j} p_{j}\left|\psi_{j}\right\rangle\left\langle\psi_{j}\right|$. Density matrices are positive operators so their eigenvalues are positive and real. For a operator $M$ we denote by $\operatorname{Tr}(M)$ the trace, or the sum of the eigenvalues, of $M$. In the case of density matrices we have $\operatorname{Tr}(\rho)=1$.

Unitary operators $U$ transform density matrices according to the rule $\rho \mapsto$ $U^{\dagger} \rho U$. A measurement $\mathcal{M}=\left\{P_{i}\right\}$ will transform the states by the rule $\rho \mapsto$ $\sum_{i} P_{i} \rho P_{i}$ in the case that the outcome is known, or by $\rho \mapsto P_{i} \rho P_{i} / \operatorname{Tr}\left(P_{i} \rho\right)$ if the outcome is known to be $i$.

Density matrices are examples of normal matrices. The spectral decomposition theorem states that every normal matrix can be decomposed as $\rho=$ $\sum_{i} \lambda_{i}\left|\phi_{i}\right\rangle\left\langle\phi_{i}\right|$, where $\left\{\left|\phi_{i}\right\rangle\right\}$ is a set of orthonormal eigenvalues of $\rho$ and $\lambda_{i}$ is the eigenvalue corresponding to $\left|\phi_{i}\right\rangle$. We say that the support of $\rho$, or $\operatorname{supp}(\rho)$, is the space spanned by the eigenvalues of $\rho$.

\section{Results}

Fix a GQFA $M$. We will be using density matrices weighted by a factor $p \in$ $[0,1]$ to describe the state of $M$ on reading some prefix $₫ w$. Let $A_{a}$ be the 
mapping $\rho \mapsto \sum_{i} P_{a, i} U_{a} \rho U_{a}^{\dagger} P_{a, i}$, and let $A_{a}^{\prime}=P_{\text {non }}\left(A_{a} \rho\right) P_{\text {non }}$. Furthermore for $w=w_{1} \ldots w_{n} \in \Sigma^{*}$, we define $A_{w}^{\prime}=A_{w_{n}}^{\prime} \ldots A_{w_{1}}^{\prime}$. Then $A_{w}^{\prime} \rho$ is a scaled density matrix such that $\operatorname{Tr}\left(A_{w}^{\prime} \rho\right)=p_{w} \operatorname{Tr}(\rho)$ where $p_{w}$ is the probability of not halting in the process of reading $w$. Let $\rho_{w}=A_{\mathbb{\Phi} w}^{\prime}\left|q_{0}\right\rangle\left\langle q_{0}\right|$. Then $\operatorname{Tr}\left(\rho_{w}\right)$ is the probability of not halting while processing $₫ w$, and the density matrix describing the machine state in the case that it has not halted is $\rho_{w} / \operatorname{Tr}\left(\rho_{w}\right)$.

We first state a technical lemma which gives an important characterization of the behaviour of a GQFA machine. It is the counterpart to Lemma 1 of [2]. This, along with its extension (Lemma 2), will be instrumental in proving the later results.

Lemma 1. For every $w \in \Sigma^{*}$ there exists a pair $E_{1}, E_{2}$ of orthonormal subspaces of $\mathbb{C}^{n}$ such that $\mathbb{C}^{n}=E_{1} \oplus E_{2}$ and for all weighted density matrices $\rho$ over $\mathbb{C}^{n}$ we have:

1. If $\operatorname{supp}(\rho) \subseteq E_{1}$, then $\operatorname{supp}\left(A_{w}^{\prime} \rho\right) \subseteq E_{1}$ and $\operatorname{Tr}\left(A_{w}^{\prime} \rho\right)=\operatorname{Tr}(\rho)$.

2. If $\operatorname{supp}(\rho) \subseteq E_{2}$, then $\operatorname{supp}\left(A_{w}^{\prime} \rho\right) \subseteq E_{2}$ and $\lim _{k \rightarrow \infty} \operatorname{Tr}\left(\left(A_{w}^{\prime}\right)^{k} \rho\right)=0$.

The $E_{1}$ and $E_{2}$ parts of the state are called the ergodic and transient parts. Suppose $M$ is in state $\rho$, and suppose that $\rho$ satisfies $\operatorname{supp}(\rho) \subseteq E_{1}$. Then $\operatorname{Tr}\left(A_{w}^{\prime} \rho\right)=\operatorname{Tr}(\rho)$ would imply that $M$ did not halt in the process of reading $w$. Thus, $M$ is behaving exactly as an LQFA. Suppose now that $M$ is in state $\rho$, then the fact $\lim _{k \rightarrow \infty} \operatorname{Tr}\left(\left(A_{w}^{\prime}\right)^{k} \rho\right)=0$ implies that the probability that $M$ does not halt after reading $w^{k}$ tends to 0 as $k \rightarrow \infty$. In general $\operatorname{supp}(\rho)$ will be partially in $E_{1}$ and partially in $E_{2}$. FAs:

This can be used to prove the following condition for recognizability by GQ-

Theorem 1. Let $M_{L}$ be the minimal automaton for $L \subseteq \Sigma^{*}$ and let $F$ be the accepting set. If there exists words $x, y, z_{1}, z_{2} \subseteq \Sigma^{*}$ and states $q_{0}, q_{1}, q_{2}$ such that $\delta\left(q_{0}, x\right)=q_{1}, \delta\left(q_{0}, y\right)=q_{2}, \delta\left(q_{1}, x\right)=\delta\left(q_{1}, y\right)=q_{1}, \delta\left(q_{2}, x\right)=\delta\left(q_{2}, y\right)=q_{2}$, $\delta\left(q_{1}, z_{1}\right) \in F, \delta\left(q_{2}, z_{1}\right) \notin F, \delta\left(q_{1}, z_{2}\right) \notin F, \delta\left(q_{2}, z_{2}\right) \in F$, then $L$ cannot be recognized by GQFA with probability $p>\frac{1}{2}$.

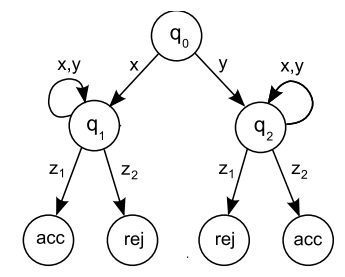

Fig. 1. The forbidden construction of Theorem 1.

We prove the theorem by using an variation of Lemma 1 to show that for any GQFA $M$ there exists words $w_{1} \in x(x \cup y)^{*}, w_{2} \in y(x \cup y)^{*}$ such that $M$ 
cannot suitably distinguish between the words $\left\{w_{i} z_{j}\right\}$. Theorem 1 can be used to show:

Theorem 2. The class of languages recognized by GQFA with bounded error is not closed under union.

In [2] it was shown that there exists languages $L$ and constants $p>\frac{1}{2}$ such that $L$ can be recognized by KWQFA with bounded probability, but not with probability $p$. Furthermore, it was demonstrated that certain properties of the minimal automaton for $L$ would imply that $L$ is not recognized with probability $p$. We will show that a similar situation holds for GQFAs.

Theorem 3. If the minimal DFA $M_{L}$ for $L$ contains states $q_{0}, q_{1}, q_{2}$, such that for some words $x, y, z_{1}, z_{2}$ we have $\delta\left(q_{0}, x\right)=\delta\left(q_{1}, x\right)=\delta\left(q_{1}, y\right)=q_{1}, \delta\left(q_{0}, y\right)=$ $\delta\left(q_{2}, y\right)=\delta\left(q_{2}, x\right)=q_{2}, \delta\left(q_{2}, z_{2}\right) \in F, \delta\left(q_{2}, z_{1}\right) \notin F$, then $L$ cannot be recognized by GQFA with probability $p>\frac{2}{3}$.

Corollary 1. There is a language $L$ which can be recognized by GQFAs with probability $p=2 / 3$, but not with $p>2 / 3$.

We note here that not all of the KWQFA lower bound results hold for GQFA. For example, it was shown that the language $a^{*} b^{*}$ can be recognized with probability $p \approx 0.68$ but not $p>7 / 9$. A stronger condition is required for GQFAs since $a^{*} b^{*}$ can be recognized by GQFA with probability $1-\varepsilon$ for any $\varepsilon>0$.

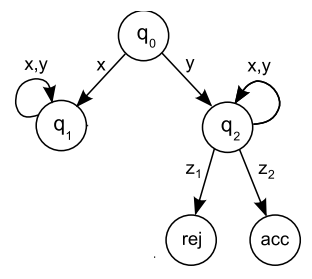

Fig. 2. The forbidden construction of Theorem 3 .

\section{Technical Results}

In this section we give a proof of Lemma 1, as well as an extension (Lemma 2).

Proof: (Lemma 1) The proof proceeds as in [2]. We first show how to do this for the case that $|w|=1$, and then we sketch how to extend it to arbitrary length words. Let $w=a$. We first construct the subspace $E_{1}$ of $\mathbb{C}^{n} . E_{2}$ will be the orthogonal complement of $E_{1}$. Let

$$
E_{1}^{1}=\operatorname{span}\left(\left\{|\psi\rangle: \operatorname{Tr}\left(A_{a}^{\prime}|\psi\rangle\langle\psi|\right)=\operatorname{Tr}(|\psi\rangle\langle\psi|)\right\}\right) .
$$


Equivalently, $E_{1}^{1}=\operatorname{span}\left\{|\psi\rangle: \operatorname{supp}\left(A_{a}(|\psi\rangle\langle\psi|)\right) \subseteq S_{\text {non }}\right\}$ where $S_{\text {non }}$ is the nonhalting subspace. We claim that $\operatorname{supp}(\rho) \in E_{1}^{1}$ implies that $\operatorname{supp}\left(A_{a}(\rho)\right) \in$ $S_{\text {non }}$. By linearity it is sufficient to show this for $\rho=|\psi\rangle\langle\psi|$. Essentially, we need to show that the condition of $|\psi\rangle$ satisfying $\operatorname{Tr}\left(A^{\prime}|\psi\rangle\langle\psi|\right)=\operatorname{Tr}(|\psi\rangle\langle\psi|)$ is closed under linear combinations. Suppose that $|\psi\rangle=\sum_{j} \alpha_{j}\left|\psi_{j}\right\rangle$, with $\left|\psi_{j}\right\rangle$ satisfying $\operatorname{supp}\left(A_{a}\left(\left|\psi_{j}\right\rangle\left\langle\psi_{j}\right|\right)\right) \in S_{\text {non }}$ and $\sum_{j}\left|\alpha_{j}\right|^{2}=1$. Then:

$$
\| \sum_{i} P_{h a l t} P_{a, i} U_{a}\left(\sum_{j} \alpha_{j}\left|\psi_{j}\right\rangle\right)\left\|^{2} \leq \sum_{i, j}\right\| \alpha_{j} P_{h a l t} P_{a, i} U_{a}\left|\psi_{j}\right\rangle \|^{2}=0
$$

and thus $\operatorname{supp}\left(A_{a}|\psi\rangle\langle\psi|\right) \in S_{\text {non }}$. Thus, for mixed states $\rho$ we have $\operatorname{supp}\left(A_{a} \rho\right) \in$ $S_{n o n}$ if and only if $\operatorname{supp}(\rho) \in E_{1}^{1}$. For general $i>2$, let:

$$
E_{1}^{i}=\operatorname{span}\left(\left\{|\psi\rangle: \operatorname{supp}\left(A_{a}|\psi\rangle\langle\psi|\right) \in E_{1}^{i-1} \wedge \operatorname{Tr}\left(A_{a}^{\prime}|\psi\rangle\langle\psi|\right)=\operatorname{Tr}(|\psi\rangle\langle\psi|)\right\}\right) .
$$

As before, for weighted density matrices $\rho$, we can interchange the condition $\operatorname{Tr}\left(A_{a}^{\prime} \rho\right)=\operatorname{Tr}(\rho)$ for $\operatorname{supp}\left(A_{a} \rho\right) \subseteq S_{\text {non }}$.

Observe that $E_{1}^{i} \subseteq E_{1}^{i+1}$ for all $i$. Since the dimension of each of these spaces is finite, there must be an $i_{0}$ such that $E_{1}^{i_{0}}=E_{1}^{i_{0}+j}$ for all $j>0$. We define $E_{1}=E_{1}^{i_{0}}$, and set $E_{2}$ to be the orthogonal complement of $E_{1}$.

It is clear that the first condition of the lemma is true for mixed states with support in $E_{1}$. For the second part, it will be sufficient to show the following claim. The claim implies that the probability with which the machine will halt while reading $a^{j}$ is bounded by a constant.

Claim. Let $j \in\left\{1, \ldots, i_{0}\right\}$. There is a constant $\delta_{j}>0$ such that for any $|\psi\rangle \in E_{2}^{j}$ there is an $l \in\{0, \ldots, j-1\}$ such that $\operatorname{Tr}\left(P_{\text {halt }} A_{a}\left(A_{a}^{\prime}\right)^{l}(|\psi\rangle\langle\psi|)\right) \geq \delta_{j}$.

Proof: We proceed by induction on $j$. Let $\mathcal{H}=\bigoplus_{k=1}^{m_{a}} \mathbb{C}^{n}$. Let $P_{k}: E_{2}^{1} \rightarrow \mathcal{H}$ be the projector into the $k$ th component of $\mathcal{H}$, and let $T_{1}: E_{2}^{1} \rightarrow \mathcal{H}$ be the function $T_{1}|\psi\rangle=\sum_{k} P_{k} P_{\text {halt }} P_{a, k} A_{a}|\psi\rangle$. Observe that $\| T_{1}|\psi\rangle \|^{2}$ is the probability of halting when $a$ is read while the machine is in state $|\psi\rangle\langle\psi|$. By the previous discussion, $\operatorname{Tr}\left(A_{a}^{\prime}|\psi\rangle\langle\psi|\right)=1-\| T_{1}|\psi\rangle \|^{2}$. Define $\left\|T_{1}\right\|=\min _{\||\psi\rangle \|=1} \| T_{1}|\psi\rangle \|$. Note that the minimum exists since the set of unit vectors in $\mathbb{C}^{n}$ is a compact space. Also, let $\delta_{1}=\left\|T_{1}\right\|^{2}$. Then $\delta_{1}>0$, otherwise there would be a vector $|\psi\rangle \in E_{2}^{1}$ such that $\operatorname{supp}\left(A_{a}|\psi\rangle\langle\psi|\right) \in S_{\text {non }}$, a contradiction.

Now assume that $\delta_{j-1}$ has been found. We need to show that, for $|\psi\rangle \in E_{2}^{j}$, either a constant sized portion of $|\psi\rangle$ is sent into the halting subspace, or it is mapped to a vector on which we can apply the inductive assumption. We construct two functions $T_{j, h a l t}, T_{j, n o n}: E_{2}^{j} \rightarrow \mathcal{H}$ defined by:

$$
\begin{aligned}
T_{j, \text { halt }}|\psi\rangle & =\sum_{k=1}^{m_{a}} P_{k} P_{\text {halt }} P_{a, k} A_{a}|\psi\rangle, \\
T_{j, \text { non }}|\psi\rangle & =\sum_{k=1}^{m_{a}} P_{k} P_{E_{2}^{j-1}} P_{n o n} P_{a, k} A_{a}|\psi\rangle .
\end{aligned}
$$


Then the quantity $\| T_{j, \text { halt }}|\psi\rangle \|^{2}$ is the probability of halting while reading $a$, and $\| T_{j, n o n}|\psi\rangle \|^{2}=\operatorname{Tr}\left(P_{E_{2}^{j-1}} A_{a}^{\prime}|\psi\rangle\langle\psi|\right)$. Note that for all vectors $|\psi\rangle \in E_{2}^{j}$ we must have either $\| T_{j, h a l t}|\psi\rangle \| \neq 0$ or $\| T_{j, \text { non }}|\psi\rangle \| \neq 0$, otherwise $|\psi\rangle$ is in $E_{1}^{j}$, a contradiction. This implies that $\left\|T_{j, \text { non }} \oplus T_{j, \text { halt }}\right\|>0$. Note also that $\left\|T_{j, \text { non }} \oplus T_{j, \text { halt }}\right\| \leq 1$.

Define $\delta_{j}=\delta_{j-1} \frac{\left\|T_{j, n o n} \oplus T_{j, \text { halt }}\right\|^{2}}{2 m_{a}}$. Take any unit vector $|\psi\rangle \in E_{2}^{j}$. Then $\|\left(T_{j, \text { non }} \oplus T_{j, \text { halt }}\right)|\psi\rangle\|\geq\| T_{j, \text { non }} \oplus T_{j, \text { halt }} \|$. Recall that the range of $T_{j, \text { non }} \oplus T_{j, \text { halt }}$ is $\bigoplus_{k=1}^{m_{a}} \mathbb{C}^{n} \oplus \bigoplus_{k=1}^{m_{a}} \mathbb{C}^{n}$. In one of these subspaces, $\left(T_{j, \text { non }} \oplus T_{j, \text { halt }}\right)|\psi\rangle$ has size at least $\frac{1}{\sqrt{2 \cdot m_{a}}}$. If it is in one of the last $m_{a}$ subspaces, corresponding to $T_{j, \text { halt }}$ part, then there is nothing further to prove. Otherwise, assume that this component is in one of the subspaces corresponding to the $T_{j, \text { non }}$ part. In particular, there is a $k$ such that $|\phi\rangle=P_{n o n} P_{a, k} A_{a}|\psi\rangle$ satisfies:

$$
\| P_{E_{2}^{j-1}}|\phi\rangle \|^{2} \geq \frac{1}{2 \cdot m_{a}} .
$$

We can split $|\phi\rangle$ into $\left|\phi_{1}\right\rangle+\left|\phi_{2}\right\rangle$, with $\left|\phi_{i}\right\rangle \in E_{i}^{j-1}$. By the inductive hypothesis, there is an $l<j-1$ such that $\operatorname{Tr}\left(P_{\text {halt }} A_{a}\left(A_{a}^{\prime}\right)^{l}\left(\left|\phi_{2}\right\rangle\left\langle\phi_{2}\right|\right)\right) \geq \delta_{j-1} \operatorname{Tr}\left(\left|\phi_{2}\right\rangle\left\langle\phi_{2}\right|\right)$. Furthermore, the first condition of the lemma implies that for every choice of $\left(k_{1}, \ldots, k_{l}\right) \in\left[m^{a}\right]^{l}$,

$$
P_{h a l t} P_{a, k_{l}} U_{a} P_{a, k_{l-1}} U_{a} \cdots P_{a, k_{1}} U_{a}\left|\phi_{1}\right\rangle=\mathbf{0} .
$$

This implies $\operatorname{Tr}\left(P_{\text {halt }} A_{a}\left(A_{a}^{\prime}\right)^{l}\left(\left|\phi_{1}\right\rangle\left\langle\phi_{1}\right|\right)\right)=0$ and $\operatorname{Tr}\left(P_{\text {halt }} A_{a}\left(A_{a}^{\prime}\right)^{l}\left(\left|\phi_{1}\right\rangle\left\langle\phi_{2}\right|\right)\right)=$ $\operatorname{Tr}\left(P_{\text {halt }} A_{a}\left(A_{a}^{\prime}\right)^{l}\left(\left|\phi_{2}\right\rangle\left\langle\phi_{1}\right|\right)\right)=0$. Together, we obtain:

$$
\begin{aligned}
& \operatorname{Tr}\left(P_{\text {halt }} A_{a}\left(A_{a}^{\prime}\right)^{l}|\phi\rangle\langle\phi|\right) \\
= & \operatorname{Tr}\left(P_{\text {halt }}\left(A_{a}^{\prime}\right)^{l}\left(\left|\phi_{1}\right\rangle\left\langle\phi_{1}|+| \phi_{1}\right\rangle\left\langle\phi_{2}|+| \phi_{2}\right\rangle\left\langle\phi_{1}|+| \phi_{2}\right\rangle\left\langle\phi_{2}\right|\right)\right) \\
= & \operatorname{Tr}\left(P_{\text {halt }} A_{a}\left(A_{a}^{\prime}\right)^{l}\left(\left|\phi_{1}\right\rangle\left\langle\phi_{1}\right|\right)\right)+\operatorname{Tr}\left(P_{\text {halt }} A_{a}\left(A_{a}^{\prime}\right)^{l}\left(\left|\phi_{1}\right\rangle\left\langle\phi_{2}\right|\right)\right) \\
& \quad+\operatorname{Tr}\left(P_{\text {halt }} A_{a}\left(A_{a}^{\prime}\right)^{l}\left(\left|\phi_{2}\right\rangle\left\langle\phi_{1}\right|\right)\right)+\operatorname{Tr}\left(P_{\text {halt }} A_{a}\left(A_{a}^{\prime}\right)^{l}\left(\left|\phi_{2}\right\rangle\left\langle\phi_{2}\right|\right)\right) \\
= & \operatorname{Tr}\left(P_{\text {halt }} A_{a}\left(A_{a}^{\prime}\right)^{l}\left(\left|\phi_{2}\right\rangle\left\langle\phi_{2}\right|\right)\right) \geq \delta_{j-1} \frac{\left\|T_{j, \text { non }} \oplus T_{j, \text { halt }}\right\|^{2}}{2 m_{a}} .
\end{aligned}
$$

This concludes the proof of the claim.

Proposition 1. Let $U_{a}$ be the unitary transformation that is applied when a is read. Then $U_{a}=U_{a}^{1} \oplus U_{a}^{2}$, where $U_{a}^{i}$ acts unitarily on subspace $E_{i}$.

Proof: By the unitarity of $U_{a}$, it is sufficient to show that $|\psi\rangle \in E_{1}$ implies $U_{a}|\psi\rangle \in E_{1}$. By definition of $E_{1},|\psi\rangle \in E_{1}$ implies that all of the vectors $P_{a, i} U_{a}|\psi\rangle$ are in $E_{1}$. But $U_{a}|\psi\rangle=\sum_{i} P_{a, i} U_{a}|\psi\rangle$, and thus $U_{a}|\psi\rangle \in E_{1}$ since $E_{1}$ is a subspace.

We are now ready to prove the second part of the lemma. We first show that $|\psi\rangle \in E_{2}$ implies $\operatorname{supp}\left(A_{a}|\psi\rangle\langle\psi|\right) \subseteq E_{2}$. Let $\left|\psi^{\prime}\right\rangle=U_{a}|\psi\rangle$. Then $A_{a}|\psi\rangle\langle\psi|=$ 
$\sum_{i}\left|\psi_{i}\right\rangle\left\langle\psi_{i}\right|$, where $\left|\psi_{i}\right\rangle=P_{a, i} U_{a}|\psi\rangle$. Split $\left|\psi_{i}\right\rangle$ into vectors $\left|\psi_{i, 1}\right\rangle+\left|\psi_{i, 2}\right\rangle$, with $\left|\psi_{i, 1}\right\rangle \in E_{1}$ and $\left|\psi_{i, 2}\right\rangle \in E_{2}$. We claim that either $\left|\psi_{i, 1}\right\rangle$ or $\left|\psi_{i, 2}\right\rangle$ are trivial vectors. Suppose $\|\left|\psi_{i, 1}\right\rangle \| \neq 0$, and consider the intersection of the image of $P_{a, i}$ in the space spanned by $\left|\psi_{i, 1}\right\rangle$ and $\left|\psi_{i, 2}\right\rangle$. Now $\left|\psi_{i, 1}\right\rangle$ implies that $U_{a}^{-1}\left|\psi_{i, 1}\right\rangle \in E_{1}$ and thus $P_{a, i}\left|\psi_{i, 1}\right\rangle \in E_{1}$, which implies $\left|\psi_{i}\right\rangle \in E_{1}$.

Now since each $\left|\psi_{i}\right\rangle$ satisfies $\left|\psi_{i}\right\rangle \in E_{1}$ or $\left|\psi_{i}\right\rangle \in E_{2}$, then we are done since the fact that the $\left|\psi_{i}\right\rangle$ 's are orthonormal and sum to $U_{a}|\psi\rangle \in E_{2}$ implies that $\left|\psi_{i}\right\rangle \in E_{2}$ for all $i$. Thus, $|\psi\rangle \in E_{2}$ implies $\operatorname{span}\left(A_{a}|\psi\rangle\langle\psi|\right) \subseteq E_{2}$.

Now supposing $\operatorname{supp}(\rho) \in E_{2}$, we can repeatedly apply the Claim to show that $\operatorname{Tr}\left(\left(A_{a}^{\prime}\right)^{k}(\rho)\right) \rightarrow 0$ as $k \rightarrow \infty$. To apply the claim to a general mixed state, we first use the spectral decomposition to show that the mixed state is equivalent to an ensemble of at most $n$ pure states.

To construct $E_{1}$ and $E_{2}$ for $w=w_{1} \ldots w_{n}$, we define $E_{1}^{0}=S_{n o n}$ and $E_{1}^{k}$ to be the set of all vectors $|\psi\rangle$ such that $\operatorname{Tr}\left(A_{w_{k} \bmod n+1}^{\prime}|\psi\rangle\langle\psi|\right)=1$ and $\operatorname{supp}\left(A_{w_{k} \bmod n+1}^{\prime}|\psi\rangle\langle\psi|\right) \in E_{1}^{k-1}$, and we follow the proof as above. The proof of the first part of the theorem and of the claim will generalize since the proof does not make use of the fact that the transformation and measurement defining $E_{1}^{j}$ is the same as that of $E_{1}^{j+1}$. Proposition 1 will apply to $w_{i}$ for all $i$.

Lemma 2. Let $M$ be an $n$-state $G Q F A$ over alphabet $\Sigma$, and let $x, y \in \Sigma^{*}$. Then there exists a pair $E_{1}, E_{2}$ of orthonormal subspaces of $\mathbb{C}^{n}$ such that $\mathbb{C}^{n}=E_{1} \oplus E_{2}$ and for all weighted density matrices $\rho$ over $\mathbb{C}^{n}$ we have:

1. If $\operatorname{supp}(\rho) \subseteq E_{1}$, then for all $w \in(x \cup y)^{*}, \operatorname{supp}\left(A_{w}^{\prime} \rho\right) \subseteq E_{1}$, and $\operatorname{Tr}\left(A_{w}^{\prime} \rho\right)=$ $\operatorname{Tr}(\rho)$.

2. If $\operatorname{supp}(\rho) \subseteq E_{2}$, then $\operatorname{supp}\left(A_{w}^{\prime} \rho\right) \subseteq E_{2}$ and for all $\varepsilon>0$ there exists a word $w \in(x \cup y)^{*}$ such that $\operatorname{Tr}\left(A_{w}^{\prime} \rho\right) \leq \varepsilon$.

Proof: This is the counterpart of Lemma 2.3 of [4]. Let $E_{1}^{w}$ be the subspace constructed as in Lemma 1. Define $E_{1}=\cap_{w \in(x \cup y)^{*}} E_{1}^{w}$, and let $E_{2}$ be the orthogonal complement of $E_{1}$.

Suppose $\operatorname{supp}(\rho) \subseteq E_{2}$. If there is a $w \in(x \cup y)^{*}$ such that $\operatorname{supp}(\rho) \subseteq E_{2}^{w}$, we can directly apply the argument from the previous lemma to show that $\operatorname{Tr}\left(\left(A_{w}^{\prime}\right)^{j} \rho\right) \rightarrow 0$ as $j \rightarrow \infty$. However such a $w$ may not exist so a stronger argument is necessary. As the application of an $A_{w}^{\prime}$ transformation can only decrease the trace of $\rho$, for any $\varepsilon$ there exists a $t \in(x \cup y)^{*}$ such that for all $w \in(x \cup y)^{*}, \operatorname{Tr}\left(A_{t}^{\prime} \rho\right)-\operatorname{Tr}\left(A_{t w}^{\prime}\right) \leq \varepsilon$. For all $i$ let $t_{i}$ be a such a string for $\varepsilon=\frac{1}{2^{i}}$. Consider the sequence $\rho_{1}, \rho_{2}, \ldots$ defined by $\rho_{i}=A_{t_{i}}^{\prime} \rho$. The set of weighted density matrices form a compact, closed space with respect to the trace metric, and so this sequence of must have a limit point $\rho$.

We claim that $\operatorname{Tr}(\rho)=0$. Suppose not. The support of $\rho$ is in $E_{2}$, so there must be some word $w \in(x \cup y)^{*}$ such that $\operatorname{Tr}\left(A_{w}^{\prime} \rho\right)<\operatorname{Tr}(\rho)$. This contradicts the assumption that $\rho$ is a limit point.

Finally we note a very simple fact that will allow us to extend impossibility results for LQFA to GQFA: 
Fact 1 Let $M$ be a GQFA. Let $E_{1}$ be the subspace defined as in Lemma 2, and suppose that the state of the machine $\rho$ on reading the $\phi$ character satisfies $\operatorname{supp}(\rho) \in E_{1}$. Then there is an LQFA $M^{\prime}$ such that, for all $w \in(x \cup y)^{*}$ the state of $M$ on reading $w$ is isomorphic to the state of $M^{\prime}$ on reading $w$.

\section{Applications}

We are now ready to apply these technical results to prove several fundamental properties of GQFAs. We begin with the proof of Theorem 1.

Proof: Suppose that $L$ satisfies the conditions of the theorem, and suppose that $M$ recognizes $L$ with probability $p>\frac{1}{2}$. By closure under left quotient, we can assume that the state $q_{0}$ in the forbidden construction is also the initial state of the minimal automaton for $L$.

Let $\rho_{w}=A_{\Phi w}^{\prime}\left|q_{0}\right\rangle\left\langle q_{0}\right|$. The basic outline of the proof is that we will use Lemma 2 to find two words $w_{1} \in x(x \cup y)^{*}, w_{2} \in y(x \cup y)^{*}$ such that $\rho_{w_{1}}$ and $\rho_{w_{2}}$ have similar output behavior. We then analyze the acceptance probabilities of the words $w_{1} z_{1}, w_{1} z_{2}, w_{2} z_{1}$, and $w_{2} z_{2}$ to arrive at a contradiction.

Let $E_{1}$ and $E_{2}$ be subspaces which meet the conditions of Lemma 2 with respect to $x$ and $y$. Note that if the support of $\rho$ is in $E_{1}, M$ will not halt while reading $w \in(x \cup y)^{*}$, and in this case $M$ can be simulated by an LQFA. Let $P_{E_{i}}$ be the projection onto subspace $E_{i}$. We claim that for all $\varepsilon>0$ there exists $u, v \in(x \cup y)^{*}$ such that $\left\|\operatorname{Tr}\left(P_{E_{1}} \rho_{x u}-P_{E_{1}} \rho_{y v}\right)\right\|_{t} \leq \varepsilon$. Suppose to the contrary that there exists $\varepsilon>0$ such that $\left\|\operatorname{Tr}\left(P_{E_{1}} \rho_{x u}-P_{E_{1}} \rho_{y v}\right)\right\|_{t}>\varepsilon$ for all $u, v$. Then there exists an LQFA which can recognize the language $x(x \cup y)^{*}$ with bounded error, contradicting the fact that LQFA is closed under inverse morphisms and cannot recognize $a \Sigma^{*}[1]$. Let $\delta=p-\frac{1}{2}$ and let $\varepsilon=\frac{\delta}{4}$.

By Lemma 2, for all $\varepsilon^{\prime}$ we can find $u^{\prime} \in(x \cup y)^{*}$ such that $\operatorname{Tr}\left(P_{E_{2}} \rho_{x u u^{\prime}}\right)<$ $\varepsilon^{\prime}$. Furthermore we can find $v^{\prime} \in(x \cup y)^{*}$ such that $\operatorname{Tr}\left(P_{E_{2}} \rho_{x u u^{\prime} v^{\prime}}\right)<\varepsilon^{\prime}$ and $\operatorname{Tr}\left(P_{E_{2}} \rho_{y v u^{\prime} v^{\prime}}\right)<\varepsilon^{\prime}$. Let $w_{1}=x u u^{\prime} v^{\prime}$ and $w_{2}=y v u^{\prime} v^{\prime}$, and let $\varepsilon^{\prime}=\frac{\delta}{4}$.

Let $p_{i, a c c}\left(p_{i, r e j}\right)$ be the probability with which $M$ accepts (rejects) while reading $w_{i}$. Furthermore let $q_{i j, a c c}\left(\operatorname{resp} q_{i j, r e j}\right)$ be the probability that $M$ accepts if the state of the machine is $\rho_{w_{1}}$ and the string $z_{j} \$$ is read. Since $\left\|\rho_{w_{1}}-\rho_{w_{2}}\right\|_{t} \leq$ $\left\|\rho_{x u}-\rho_{y v}\right\|_{t}=\frac{\delta}{2} \leq \varepsilon, q_{1 j, a c c}$ (and likewise $q_{1 j, r e j}$ ) can be different from $q_{2 j, a c c}$ by a factor of at most $\frac{\delta}{2}$. As a consequence, one of the words $w_{1} z_{1}, w_{1} z_{2}, w_{2} z_{1}$, or $w_{2} z_{2}$ must not be classified correctly. Suppose, for instance that $w_{1} z_{1}, w_{1} z_{2}$, and $w_{2} z_{1}$ are classified correctly. Since $q_{11, r e j}$ differs from $q_{21, \text { rej }}$ by a factor of at most $\frac{\delta}{2}$, the fact that $w_{1} z_{1}$ is accepted and $w_{2} z_{1}$ is rejected implies that

$p_{2, r e j}>p_{1, r e j}+\delta$. since $q_{12, r e j}$ differs from $q_{22, r e j}$ by at most a factor of $\frac{\delta}{2}$, will be rejected with probability greater than $1-p$, a contradiction. The other cases are similar.

We now apply Theorem 1 to prove nonclosure under union.

Proof: (Theorem 2) Let $A, B_{0}, B_{1}$ be languages over $\Sigma=\{a, b\}$ defined as follows. Let $A=\left\{w:|w|_{a} \bmod 2=0\right\}, B_{0}=(a a)^{*} b \Sigma^{*}$, and $B_{1}=a(a a)^{*} b \Sigma^{*}$. 
Finally, let $L_{1}=\left(\bar{A} \cap a^{*}\right) \cup\left(A \cap B_{1}\right)$, and let $L_{2}=\left(A \cap a^{*}\right) \cup\left(\bar{A} \cap B_{0}\right)$. The union $L_{1} \cup L_{2}$ consists of the strings containing either no $b$ 's or an odd number of $a$ 's after the first $b$.

In Theorem 3.2 of [4], the languages $L_{1}$ and $L_{2}$ were shown to be recognizable by KWQFAs with probability of correctness $2 / 3$, thus they can also be recognized by GQFA with this probability of correctness. The constructions rely on the fact that we allow the machine to halt an accept before the end. On the other hand, the minimal automaton of $L_{1} \cup L_{2}$ contains the forbidden construction of Theorem 1.

We now use a similar technique to prove Theorem 3.

Proof: (Theorem 3) Suppose that the GQFA $M$ recognizes $L$ with probability $p>2 / 3$. Since $q_{2} \neq q_{3}$ and by closure under complement, there exists a word $z_{3}$ such that $x z_{3} \in L$ and $y z_{3} \notin L$. We can also assume by closure under left quotient that $q_{1}$ is the initial state. As in Lemma 2, split $\mathbb{C}^{n}$ into subspaces $E_{1}$ and $E_{2}$ with respect to $x$ and $y$.

For all $\varepsilon$, we can find $w_{1} \in x(x \cup y)^{*}$ and $w_{2} \in y(x \cup y)^{*}$ such that $\| \rho_{w_{1}}-$ $\rho_{w_{2}} \|_{t} \leq \varepsilon, \operatorname{Tr}\left(P_{E_{2}} \rho_{w}\right)<\varepsilon, \operatorname{Tr}\left(P_{E_{2}} \rho_{w}\right)<\varepsilon$. let $p_{i}$ be the probability that $M$ rejects while reading $w_{i}$, and let $p_{i 3}$ be the probability of rejecting when $M$ is in state $q_{i}$ and reads $z_{3}$. By setting $\varepsilon$, the difference between $p_{13}$ and $p_{23}$ can be made arbitrarily small, so that $p_{1}+p_{13} \leq(1-p)<1 / 3$ and $p_{2}+p_{23} \geq p>2 / 3$ imply that $p_{2}-p_{1}>1 / 3$. Thus $M$ rejects while reading $w_{2}$ with probability greater than $1 / 3$, contradicting the assumption that $w_{2} z_{2}$ is accepted with probability greater than $2 / 3$.

Note that Theorem 3 implies that the GQFAs recognizing $L_{1}$ and $L_{2}$ described above achieve the optimal probability of correctness. This proves the corollary to Theorem 3 .

\section{Open Problems}

We have shown that several but not all of the known lower proofs for KWQFA can be adapted to the case of GQFA. Several other KWQFA lower bounds were shown in $[4,3]$, and we can further clarify the relationship between the two models by identifying which of these results extend to GQFAs.

While we have mentioned the existence of languages which can be recognized with probability $p$ by GQFA but not by KWQFA, it is still not known whether there the class of languages recognized by bounded error by GQFA is strictly larger than the class recognized by KWQFA. We conjecture that the language class is indeed larger and that a proof would involve the fact that the probability with which KWQFAs can recognize $\Sigma^{*} a_{1} \Sigma^{*} \ldots a_{k} \Sigma^{*}$ tends to $1 / 2$ as $k \rightarrow \infty$. 


\section{References}

1. Andris Ambainis, Martin Beaudry, Marats Golovkins, Arnolds Kikusts, Mark Mercer, and Denis Thérien. Algebraic results on quantum automata. Theory of Computing Systems, 38:165-188, 2006.

2. Andris Ambainis and Rusins Freivalds. 1-way quantum finite automata: strengths, weaknesses and generalizations. In 39th Annual Symposium on Foundations of Computer Science, pages 332-341. IEEE Computer Society Press, 1998.

3. Andris Ambainis and Arnolds Kikusts. Exact results for accepting probabilities of quantum automata. Theoretical Computer Science, 295(1-3):3-25, February 2003.

4. Andris Ambainis, Arnolds Ķikusts, and Māris Valdats. On the class of languages recognizable by 1-way quantum finite automata. In Proceedings of the 18th Annual Symposium on Theoretical Aspects of Computer Science, volume 2010 of Lecture Notes in Computer Science, pages 75-86, 2001.

5. C. H. Bennett. Logical reversibility of computation. IBM Journal of Research and development 6, pages 525-532, 1973.

6. Ethan Bernstein and Umesa Vazirani. Quantum complexity theory. SIAM Journal of Computing, 26(5):111-1473, 1997.

7. Alberto Bertoni, Carlo Mereghetti, and Beatrice Palano. Quantum computing: 1-way quantum automata. In Developments in Language Theory, volume 2710 of Lecture Notes in Computer Science. Springer, 2003.

8. Alex Brodsky and Nicholas Pippenger. Characterizations of 1-way quantum finite automata. SIAM Journal on Computing, 31(5):1456-1478, October 2002.

9. Marats Golovkins and Jean-Éric Pin. Varieties generated by certain models of reversible finite automata. In Proceedings of COCOON 2006, volume 4112 of Lecture Notes in Computer Science, pages 83-93, 2006.

10. Attila Kondacs and John Watrous. On the power of quantum finite state automata. In 38th Annual Symposium on Foundations of Computer Science, pages 66-75. IEEE Computer Society Press, 20-22 October 1997.

11. Cris Moore and Jim Crutchfield. Quantum automata and quantum grammars. Theoretical Computer Science, 237(1-2):275-306, 2000.

12. Ashwin Nayak. Optimal lower bounds for quantum automata and random access codes. In 40th Annual Symposium on Foundations of Computer Science (FOCS '99), pages 369-377, Washington - Brussels - Tokyo, 1999.

13. Ashwin Nayak and Julia Salzman. On communication over an entanglementassisted quantum channel. In Proceedings of the Thirty-Fourth Annual ACM Symposium on the Theory of Computing, pages pages 698-704, 2002.

14. Michael Nielsen and Isaac Chuang. Quantum Computation and Quantum Information. CUP, 2000.

15. Jean-Éric Pin. BG=PG, a success story. In John Fountain, editor, NATO Advanced Study Institute Semigroups, Formal Languages, and Groups, pages 33-47. Kluwer Academic Publishers, 1995.

16. Michael Rabin. Probabilistic automata. Information and Control, 6(3):230-245, September 1963.

17. Andy Yao. Quantum circuit complexity. In Proceedings of the 36th annual FOCS, pages 352-361, 1993. 